\title{
El ambiente laboral como fuente de exposición a humo de tabaco ambiental: Estudio en trabajadores de bares y restaurantes de Santiago, Chile
}

\author{
PAULINA ACEITUNO ${ }^{1}$, VERÓNICA IGLESIAS ${ }^{1, a}$, MARCIA ERAZO $^{2, \mathrm{~b}}$, \\ ANDREA DROPPELMANN ${ }^{3, c}$, CECILIA ORELLANA ${ }^{1, a}$, ANA NAVAS-ACIÉN $^{4}$
}

\section{The work environment as a source of exposure to secondhand smoke: a study in workers of bars and restaurants of Santiago, Chile}

Background: Secondhand smoke (SHS) is an established cause of morbidity and mortality among non-smokers. The workplace is an important source of exposure, especially among workers of restaurants, bars and nightclubs. Aim: To estimate the daily occupational exposure to SHS among non-smoking workers of bars and restaurants of Santiago, Chile. Material and Methods: Environmental vapor-phase nicotine was measured for 95 non-smoking workers of bars and restaurants of Santiago, using passive personal samplers during a daily work shift and outside the workplace. Results: The median occupational exposure to air nicotine was $9.18 \mu \mathrm{g} / \mathrm{m}^{3}\left(P_{25}{ }_{7} P_{75}\right.$ $\left.3.15-25.67 \mu \mathrm{g} / \mathrm{m}^{3}\right)$. Higher concentrations were found among workers of places with no smoking restrictions $\left(22.72 \mu \mathrm{g} / \mathrm{m}^{3} ; P_{25}-P_{75} 5.73-34.85 \mu \mathrm{g} / \mathrm{m}^{3}\right)$, bar workers (20.75 $\left.\mu \mathrm{g} / \mathrm{m}^{3}, P_{25}-P_{75} 5.03-44.67 \mu \mathrm{g} / \mathrm{m}^{3}\right)$, waiters $\left(20.57 \mu \mathrm{g} / \mathrm{m}^{3}, P P_{25}-P_{75} 5.66-42.73 \mu \mathrm{g} / \mathrm{m3}\right)$ and bartenders $\left(10.37 \mu \mathrm{g} / \mathrm{m}^{3}, P_{25}-P_{75} 9.75-25.67 \mu \mathrm{g} / \mathrm{m}^{3}\right)$. The median concentration of nicotine outside the workplace was $1.79 \mu \mathrm{g} / \mathrm{m}^{3}\left(P_{25}-P_{75} 1.02-3.00 \mu \mathrm{g} / \mathrm{m}^{3}\right)$. Occupational exposure was 4.77 times higher compared to the non-work exposure. Conclusions: Workers of bars and restaurants are exposed to high levels of SHS at the workplace. Moreover, occupational exposure among these non-smoking workers is, in most cases, the main source of daily exposure to this pollutant.

(Rev Med Chile 2010; 138: 1517-1523).

Key words: Confined spaces; Nicotine; Smoking.

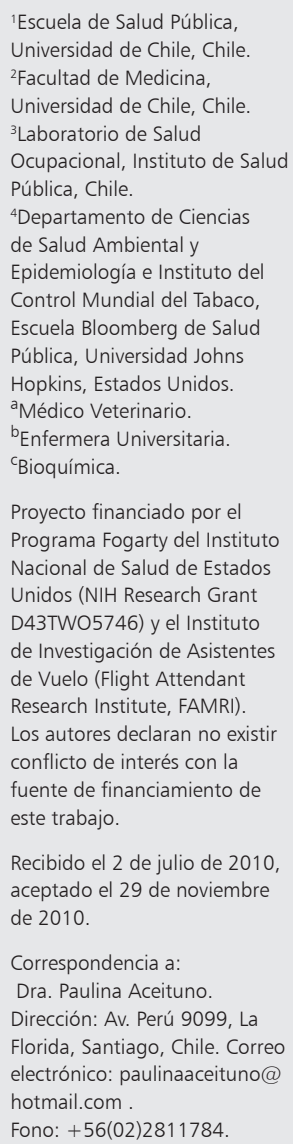

E 1 tabaquismo es una epidemia a nivel mundial cuyas consecuencias no sólo afectan a fumadores. El humo de tabaco ambiental (HTA), definido como el humo en suspensión presente en un ambiente donde alguien ha fumado, es un contaminante al que también se ven expuestos quienes optan por no fumar, denominados fumadores involuntarios o pasivos ${ }^{1,2}$. El HTA está constituido por más de 4.000 sustancias, entre ellas más de 50 carcinógenos reconocidos, por lo que ha sido calificado como un carcinógeno en humanos para el cual no existen niveles seguros de exposición ${ }^{3,4}$. La nicotina, al ser un constituyente específico del HTA, es el marcador ambiental más utilizado ${ }^{1,4}$.

Diversos estudios señalan que el HTA es el contaminante más importante al interior de viviendas y lugares de trabajo ${ }^{1,5-7}$. Numerosos trabajos han reportado altos niveles de exposición ocupacional, llegando a superar en 10 veces los niveles de exposición a HTA al interior de hogares ${ }^{1,8}$. Algunos 
investigadores consideran a trabajadores de bares y restaurantes entre las categorías ocupacionales más expuestas ${ }^{6}$. En la revisión llevada a cabo por López y Tovar sobre exposición a HTA en restaurantes, hoteles y cantinas, trabajadores de bares y restaurantes estarían expuestos a niveles de HTA hasta 6,1 y 2,0 veces más altos, respectivamente, en comparación con trabajadores de oficinas; y hasta 4,4 y 1,5 veces más, respectivamente, en comparación con los quienes conviven con un fumador en el hogar 6 .

Diversos estudios han asociado la exposición laboral a HTA con mayor riesgo de cáncer pulmonar $^{9,10}$, enfermedad coronaria ${ }^{11,12}$, asma ${ }^{13}$, así como un mayor número de consultas médicas, uso de fármacos y ausentismo laboral por problemas de salud, con el consiguiente costo para los servicios de salud, empleador y empleado ${ }^{14,15}$.

En Chile, la Ley 20.105 que regula el consumo y publicidad relacionados con el tabaco, permite el consumo de tabaco en lugares públicos como bares, restaurantes, pubs, discotecas, casinos de juegos o cabarets bajo ciertas restricciones según superficie de local y tipo de patente ${ }^{16}$. Respecto a la exposición laboral a HTA en estos locales, el Decreto Supremo No 594 que aprueba el Reglamento sobre Condiciones Sanitarias y Ambientales Básicas en los Lugares de Trabajo en Chile, no hace referencia al HTA como contaminante y, por lo tanto, no existe ninguna regulación respecto a esta exposición en trabajadores ${ }^{17}$.

El objetivo de este estudio fue determinar la exposición laboral diaria a HTA en trabajadores no fumadores de bares y restaurantes de Santiago y evaluar su asociación con determinantes sociodemográficos y relacionados con el trabajo. A su vez, se estimó la exposición diaria a HTA fuera del lugar de trabajo para comparar la exposición laboral diaria a este contaminante con respecto a la exposición diaria en otros ambientes.

\section{Material y Método}

Estudio enmarcado dentro del proyecto de investigación "Exposición a tabaco ambiental en lugares de uso público y su asociación con niveles de nicotina de sus trabajadores", realizado en conjunto por la Escuela de Salud Pública y Facultad de Medicina de la Universidad de Chile, el Instituto de Salud Pública de Chile y la Escuela de
Salud Pública de la Universidad de Johns Hopkins, Estados Unidos ${ }^{18}$.

Este estudio transversal se llevó a cabo entre abril y agosto de 2008 en Santiago, Chile. De la población de trabajadores no fumadores de bares y restaurantes de la ciudad se obtuvo, a través de un muestreo no probabilístico de locales y trabajadores, una muestra de 95 trabajadores (tamaño muestral usado en el estudio primario), considerando un error de estimación de $0,95 \mu \mathrm{g} / \mathrm{m}^{3}$ de nicotina, una desviación estándar de $4,7 \mu \mathrm{g} / \mathrm{m}^{319} \mathrm{y}$ un nivel de confianza de $95 \%$ en un test de 2 colas.

La variable resultado fue la exposición diaria a HTA la que se midió usando monitores individuales de difusión pasiva para nicotina siguiendo el protocolo desarrollado por Hammond ${ }^{20}$. La determinación de nicotina se realizó en el Laboratorio de Medición de Exposiciones Ambientales perteneciente a la Escuela de Salud Pública de Bloomberg, Universidad Johns Hopkins, utilizando cromatografía gaseosa con detector selectivo nitrógeno-fósforo ${ }^{20}$. Para estimar la concentración media, la nicotina obtenida de cada filtro $(\mu \mathrm{g})$ se estandarizó por el volumen de aire $\left(\mathrm{m}^{3}\right)$ filtrado durante el período de medición. Para el filtro utilizado, se estima un volumen de filtración de $25 \mathrm{ml} /$ $\mathrm{min}^{20}$. Esta técnica ha sido validada en condiciones experimentales y usada en estudios de monitoreo ambiental y personal ${ }^{20-23}$. El uso de monitores personales, comparado con monitores fijados en un lugar del local, refleja más directamente exposición individual a HTA al considerar la circulación por ambientes con diferentes niveles de exposición ${ }^{24,25}$.

A cada trabajador se le entregó un monitor para medir exposición a HTA durante la jornada laboral, y un segundo para medir exposición a HTA fuera del lugar del trabajo (desde que salió de su trabajo hasta que volvió a éste el día siguiente).

El límite de detección de nicotina fue de 0,09 $\mu \mathrm{g} / \mathrm{m}^{3}$. De los monitores usados fuera del trabajo, $3,4 \%$ presentaron concentraciones de nicotina bajo este valor. En estos casos se asignó un valor equivalente a la mitad del límite de detección. Ningún monitor laboral estuvo bajo este límite.

Como variables independientes se incluyeron: variables relacionadas con el trabajo (ocupación dentro del local, día de la semana monitoreado, jornada durante ese día) y variables relacionadas con el local (tipo de local, estatus de fumador, superficie del local habilitado al público y existencia de sistemas de extracción del humo). Para carac- 
Ambiente laboral y exposición a humo de tabaco - P. Aceituno et al

terizar la muestra se analizaron además variables sociodemográficas (sexo, edad, escolaridad, número de días semanales trabajados, horas diarias trabajadas). Las variables sociodemográficas y aquellas relacionadas con el trabajo se obtuvieron a través de un cuestionario aplicado a cada trabajador. Información respecto al local fue obtenida a través de un cuestionario aplicado al dueño/ administrador de cada recinto.

El análisis estadístico se realizó usando el programa Stata $10.0^{\circledR}$. En el análisis descriptivo se calcularon medidas de tendencia central, dispersión y tablas de frecuencia. Posteriormente, se realizó un análisis bivariado entre exposición laboral a HTA y las diferentes variables independientes identificadas. La prueba estadística Kruskal Wallis fue usada para variables independientes categóricas y se utilizó la prueba de Mann Whitney en caso de variables independientes dicotómicas. Por último, se calculó la relación existente entre exposición laboral y no laboral a HTA, y se utilizó la prueba de Wilcoxon para muestras pareadas para determinar la existencia de diferencias significativas de exposición entre ambos ambientes. Un nivel de significación de 5\% fue usado en todos los casos.

El protocolo de investigación y consentimiento informado del estudio primario fue revisado y aprobado por el Comité de Ética de la Facultad de Medicina de la Universidad de Chile. Se obtuvo el consentimiento informado por parte del dueño/ administrador del local y de los trabajadores que aceptaron participar.

\section{Resultados}

De 57 locales inicialmente contactados, 7 (12,3\%) fueron excluidos por contar con menos de 2 trabajadores no fumadores. En 10 locales $(17,5 \%)$ no se obtuvo una respuesta formal por parte del dueño/administrador y en 15 locales $(28,1 \%)$ se rechazó la invitación a participar. En los 25 locales restantes se reclutó a 97 trabajadores no fumadores con una mediana de 3 trabajadores por local (mín-máx 2-12). Se obtuvo un rechazo de 2,1\%. De los 95 trabajadores que aceptaron participar, se recuperaron 89 monitores usados durante la jornada laboral (93,7\%); sólo estos individuos fueron incluidos en el análisis. Los 6 trabajadores excluidos trabajaban en su mayoría en locales que permitían fumar sin restricciones
$(83,3 \%)$, en bares $(66,7 \%)$ y se ocupaban como meseros $(66,7 \%)$.

En las Tablas 1 y 2 se detallan, respectivamente, las características de los locales reclutados y las características sociodemográficas de los participantes.

La mediana de exposición laboral a nicotina fue $9,18 \mu \mathrm{g} / \mathrm{m}^{3}\left(\mathrm{P}_{25}-\mathrm{P}_{75} 3,15-25,67 \mu \mathrm{g} / \mathrm{m}^{3}\right)$. Respecto a la exposición laboral a HTA (Tabla 3), sólo se observaron diferencias significativas en relación al estatus del local (permitido fumar, prohibido fumar o áreas mixtas), tipo de local y ocupación

Tabla 1. Caracterización de los locales

\begin{tabular}{|lcc|}
\hline Variable & n & \% \\
\hline $\begin{array}{l}\text { Tipo de local } \\
\text { Bar }\end{array}$ & 13 & 52,0 \\
$\quad$ Restaurante & 12 & 48,0 \\
Estatus del local & & \\
$\quad$ Sólo fumadores & 9 & 36,0 \\
$\quad$ No fumadores & 2 & 8,0 \\
Mixto & 14 & 56,0 \\
Superficie del local 1,2 & & \\
$\quad$ Menor a 100 m² & 2 & 10,5 \\
Mayor a 100 m² & 17 & 89,5 \\
Sistema de ventilación & & \\
Si & 23 & 92,0 \\
No & 2 & 8,0 \\
\hline
\end{tabular}

${ }^{1}$ Metros cuadrados destinados a público. ${ }^{2}$ Sólo 19 (76\%) de los locales aportó el dato.

Tabla 2. Características socio-demográficas de los participantes

\begin{tabular}{|c|c|c|c|c|}
\hline Variable & $n$ & $\%$ & Mediana & Min-Max \\
\hline Edad (años) & & & 30 & $17-67$ \\
\hline $\begin{array}{l}\text { Sexo } \\
\text { Hombres } \\
\text { Mujeres }\end{array}$ & $\begin{array}{l}61 \\
28\end{array}$ & $\begin{array}{l}68,5 \\
31,5\end{array}$ & & \\
\hline $\begin{array}{l}\text { Educación } \\
\text { Básica } \\
\text { Media } \\
\text { Superior }\end{array}$ & $\begin{array}{r}8 \\
38 \\
43\end{array}$ & $\begin{array}{r}9,0 \\
42,7 \\
48,3\end{array}$ & & \\
\hline $\begin{array}{l}\text { Días a la } \\
\text { semana } \\
\text { trabajados }\end{array}$ & & & 6 & $1-7$ \\
\hline $\begin{array}{l}\text { Horas día } \\
\text { trabajadas }\end{array}$ & & & 9 & $6-15$ \\
\hline
\end{tabular}


Tabla 3. Exposición individual a nicotina ambiental en el trabajo $\left(\mu \mathrm{g} / \mathrm{m}^{3}\right)$, según características del local, ocupación y jornada laboral

\begin{tabular}{|c|c|c|c|c|c|}
\hline & n (\%) & Mediana & $\mathbf{P}_{25}-\mathbf{P}_{75}$ & Min-Max & Valor p \\
\hline Tipo del local* & & & & & $p<0,001$ \\
\hline Bar & $58(65,2)$ & 20,75 & $5,03-44,67$ & $0,81-186,49$ & \\
\hline Restaurante & $31(34,8)$ & 3,77 & $2,21-7,70$ & $0,88-13,70$ & \\
\hline Estatus del local** & & & & & $p<0,01$ \\
\hline Sólo fumadores & $32(36,0)$ & 22,72 & $5,73-34,85$ & $0,91-186,49$ & \\
\hline No fumadores & $4(4,5)$ & 3,96 & $3,10-5,09$ & $2,43-\quad 6,02$ & \\
\hline Mixto & $53(59,5)$ & 5,37 & $2,47-13,87$ & $0,81-125,16$ & \\
\hline Superficie local $1, *$ & & & & & $p=0,87$ \\
\hline Menor $100 \mathrm{~m}^{2}$ & $4(6,0)$ & 12,80 & $3,54-59,20$ & $2,08-97,84$ & \\
\hline Mayor 100 m² $^{2}$ & $63(94,0)$ & 9,82 & $3,27-27,13$ & $0,81-125,16$ & \\
\hline Sistema ventilación* & & & & & $p=0,18$ \\
\hline $\mathrm{Si}$ & $85(95,5)$ & 6,73 & $2,73-25,17$ & $0,81-186,49$ & \\
\hline No & $4(4,5)$ & 20,43 & $11,82-30,13$ & $11,28-31,75$ & \\
\hline Ocupación** & & & & & $p<0,001$ \\
\hline Dueño/Administ & $8 \quad(9,0)$ & 3,90 & $1,83-6,39$ & $1,33-11,59$ & \\
\hline Barman & $9(10,1)$ & 10,37 & $9,75-25,67$ & $2,47-125,16$ & \\
\hline Meseros & $45(50,5)$ & 20,57 & $5,66-42,73$ & $0,82-186,49$ & \\
\hline Cocina & $20(22,5)$ & 3,41 & $2,01-7,86$ & $0,81-47,00$ & \\
\hline Otros & $7 \quad(7,9)$ & 2,72 & $0,91-5,03$ & $0,88-62,76$ & \\
\hline Jornada laboral* & & & & & $p=0,08$ \\
\hline 07:00 a 17:00 & $16(18,0)$ & 4,88 & $3,30-10,64$ & $0,81-24,52$ & \\
\hline $17: 00$ a 07:00 & $73(82,0)$ & 9,86 & $2,73-31,75$ & $0,88-186,49$ & \\
\hline Día de la semana* & & & & & $p=0,70$ \\
\hline Domingo a Miércoles & $38(42,7)$ & 6,20 & $2,72-21,11$ & $1,34-125,16$ & \\
\hline Jueves a Sábado & $51(57,3)$ & 9,82 & $3,36-27,95$ & $0,81-186,49$ & \\
\hline
\end{tabular}

${ }^{1}$ Metros cuadrados abiertos al público. *Prueba Mann Whitney. ** Prueba Kruskall Wallis.

dentro del local. Los niveles de nicotina fueron mayores entre trabajadores de locales sin restricciones para fumar (mediana $22,72 \mu \mathrm{g} / \mathrm{m}^{3}$ ) y con áreas mixtas (mediana $5,37 \mu \mathrm{g} / \mathrm{m}^{3}$ ), comparado con locales donde no se permitía fumar (mediana $\left.3,96 \mu \mathrm{g} / \mathrm{m}^{3}\right)(\mathrm{p}<0,01)$. En relación al tipo de local, trabajadores de restaurantes presentaron niveles de exposición significativamente más bajos en relación a trabajadores de bares (valor $\mathrm{p}<0,001$ ).

Según la ocupación dentro del local, meseros (mediana 20,57 $\mu \mathrm{g} / \mathrm{m}^{3}$ ) presentaron niveles más altos en comparación al resto de las ocupaciones $(\mathrm{p}<0,01)$.

Respecto a la exposición a HTA fuera del lugar de trabajo, se recuperaron 88 monitores $(98,9 \%)$ obteniéndose una mediana de concentración de nicotina de $1,79 \mu \mathrm{g} / \mathrm{m}^{3}\left(\mathrm{P}_{25}-\mathrm{P}_{75} 1,02-3,00 \mu \mathrm{g} / \mathrm{m}^{3}\right)$. $\mathrm{Al}$ analizar la relación existente entre exposición laboral y no laboral a HTA, en cada trabajador, se observó una mediana de exposición 4,77 $\left(\mathrm{P}_{25}-\mathrm{P}_{75}\right.$ $1,72-17,32)$ veces mayor en el lugar de trabajo, comparado con la exposición diaria en otros ambientes, diferencia que resultó ser estadísticamente significativa $(\mathrm{n}=88 ; \mathrm{p}<0,001)$.

\section{Discusión}

Los resultados de este estudio evidencian altos niveles de exposición a HTA en trabajadores no fumadores de bares y restaurantes de Santiago, especialmente en locales donde está permitido fumar sin restricción, en bares y en trabajadores en contacto directo con el público. Más aun, los resultados muestran que el lugar de trabajo constituye, en la mayoría de los casos, la principal fuente 
diaria de exposición, resultados concordantes con lo descrito en la literatura.

El reporte del Surgeon General sobre exposición a HTA en Estados Unidos, recopila resultados de diversos estudios, usando diferentes técnicas y tiempos de monitoreo, que muestran concentraciones de nicotina entre 3,4 y $13,8 \mu \mathrm{g} / \mathrm{m}^{3}$ en restaurantes y entre 7,4 y $65,5 \mu \mathrm{g} / \mathrm{m}^{3}$ en bares ${ }^{26}$. A su vez, en el trabajo llevado a cabo por Bergman et al en diversos centros nocturnos en Estados Unidos, usando monitores personales y mediciones seriadas durante una semana, se obtuvieron concentraciones de nicotina entre 27,8 y $50,2 \mu \mathrm{g} /$ $\mathrm{m}^{3-23}$. Niveles igualmente altos fueron reportados en el estudio multicéntrico realizado en diferentes ciudades europeas por Nebot et al. Los niveles de nicotina ambiental en restaurantes, usando monitores fijos por un período de 2 días, variaron entre 0,01 y $18,0 \mu \mathrm{g} / \mathrm{m}^{3}$, mientras que los valores encontrados en bares y discos, con monitores personales usados por 4 horas, fluctuaron entre 19 a $122 \mu \mathrm{g} /$ $\mathrm{m}^{3-21}$. En el único estudio multicéntrico llevado a cabo en Latinoamérica, realizado por Navas-Acien et al el 2004, la concentración mediana de nicotina medida durante 7 días fue $1,24 \mu \mathrm{g} / \mathrm{m}^{3}\left(\mathrm{P}_{25}-\mathrm{P}_{75}\right.$; $\left.0,41-2,48 \mu \mathrm{g} / \mathrm{m}^{3}\right)$ en restaurantes y $3,65 \mu \mathrm{g} / \mathrm{m}^{3}\left(\mathrm{P}_{25}{ }^{2}\right.$ $\left.\mathrm{P}_{75} ; 1,55-5,12 \mu \mathrm{g} / \mathrm{m}^{3}\right)$ bares $^{22,27}$. Específicamente en Chile, el estudio de Navas-Acien encontró niveles de $2,08 \mu \mathrm{g} / \mathrm{m}^{3}$ de nicotina en restaurantes de Santiago $\left(\mathrm{P}_{25}-\mathrm{P}_{75} ; 1,08-3,56 \mu \mathrm{g} / \mathrm{m}^{3}\right)$, y $3,33 \mu \mathrm{g} / \mathrm{m}^{3}$ en bares $\left(\mathrm{P}_{25}-\mathrm{P}_{75} ; 2,06-4,82 \mu \mathrm{g} / \mathrm{m}^{3}\right)^{22-27}$.

Una diferencia entre el estudio de Navas-Acien et al y el presente trabajo, es que el primero realizó monitoreos ambientales por períodos continuos de 7 días (siendo presumible una menor emisión de HTA durante períodos en los cuales los locales estuvieron cerrados al público), frente al monitoreo personal del presente estudio, exclusivamente en horas de atención al público. Esta diferencia metodológica podría explicar, en parte, las diferencias encontradas entre ambos trabajos.

Se debe destacar que aunque las concentraciones de nicotina fueron mayores en los locales donde se podía fumar sin restricción, comparado con locales con áreas para fumadores y no fumadores, un $25 \%$ de los trabajadores de locales con áreas mixtas presentaron niveles mayores o iguales a $13,87 \mu \mathrm{g} / \mathrm{m}^{3}$. En relación a lo anterior, diversos estudios han mostrado lo ineficaz de la separación física de ambientes fumadores y no fumadores para evitar la contaminación de espacios destina- dos a no fumadores; además desde un punto de vista laboral, no protegería a los trabajadores obligados a transitar por ambos sectores ${ }^{28-31}$. Respecto a la detección de nicotina en locales donde no estaba permitido fumar (aunque significativamente menor comparada con el resto de los locales), lo pequeño del tamaño muestral impide un mayor análisis siendo necesario considerar la posible exposición en las zonas de descanso, colación, etc, y/o al incumplimiento de la norma por parte de los clientes y/o trabajadores.

Además de los altos niveles asociados a trabajadores directamente expuestos al público, llama la atención los altos niveles encontrados en trabajadores de la cocina, posiblemente debido a la comunicación de este espacio con el resto del local, la ausencia de barreras efectivas de separación, y la facilidad de difusión del humo de tabaco a través del ambiente. Por último es interesante analizar la ausencia de diferencias significativas de exposición entre locales con y sin sistemas de ventilación y/o dispositivos de extracción de aire al exterior. Aunque el tamaño muestral del último grupo dificulta mayores inferencias, los hallazgos son consistentes con la demostrada inefectividad de los sistemas de ventilación ${ }^{31}$. La ley actual sólo señala la obligación de contar con sistemas de extracción de aire al exterior, sin definir algún estándar al respecto ${ }^{16}$.

El presente trabajo constituye el primer estudio en Chile enfocado en cuantificar exposición laboral a HTA y espera aportar una base para futuros estudios en el área de salud ocupacional. Fortalezas importantes incluyen el uso de un marcador específico de exposición a HTA, la comparación entre exposición laboral y fuera del trabajo, el uso de un protocolo establecido, y sistemas de control y garantía de calidad en el análisis de laboratorio.

En referencia a las limitaciones del estudio, el uso de un muestreo por conveniencia pudiera afectar la representatividad de la población de locales de este rubro. Debido a la alta tasa de rechazo a participar en el estudio, es plausible plantear que los locales que aceptaron participar tuvieran menores niveles de tabaquismo comparado con aquellos que rechazaron participar y que los resultados subestiman los niveles de HTA en estos últimos. Por otro lado, el muestreo se realizó durante el otoño e invierno, siendo posible que las bajas temperaturas incidieran en una menor ventilación a través de puertas y ventanas $\mathrm{y}$, con 
ello, a niveles más altos de HTA al interior de los locales. Sin embargo, en el mencionado estudio de Navas-Acien et al, no se observaron diferencias significativas, en los niveles de HTA, entre países latinoamericanos con diferencias climáticas considerables ${ }^{22}$.

Finalmente, el uso de mediciones puntuales impide evaluar posibles diferencias de exposición entre distintos días de la semana. La inclusión de variables como día de semana monitoreado y jornada dentro del día tuvo por finalidad considerar este hecho, pero la realización de monitoreos seriados es probablemente una mejor forma de controlar este potencial sesgo.

En conclusión, los trabajadores de bares y restaurantes analizados se encuentran expuestos a niveles preocupantes de HTA siendo el lugar de trabajo, en la mayoría de los casos, la principal fuente de exposición diaria a este contaminante. Por consiguiente, queda en evidencia que la Ley de tabaco vigente en Chile no es suficiente para proteger a este sector laboral, de un factor de riesgo para la salud innecesario y evitable, siendo necesario cambios orientados a eliminar la exposición a HTA en todos los lugares públicos, incluyendo este tipo de locales.

Agradecimientos: Agradecemos al Dr. Patrick Breysse y a su equipo su importante labor en el análisis de los datos de nicotina ambiental.

\section{Referencias}

1. U.S. Department of Health and Human Services. The Health Consequences of Smoking: A Report of the Surgeon General. Atlanta, GA: U.S. Department of Health and Human Services, Centers for Disease Control and Prevention, National Center for Chronic Disease Prevention and Health Promotion, Office on Smoking and Health, 2004. Disponible en: http://www.cdc.gov/ tobacco/data_statistics/sgr/2004/complete_report/index. htm [Consultado el 2 de Marzo del 2010].

2. California Environmental Protection Agency. Office of Environmental Health Hazard Assessment. Health effects of exposure to environmental tobacco smoke. Final report. September 1997. Disponible en: http:// www.oehha.org/air/environmental_tobacco/finalets. html [Consultado el 2 de Marzo del 2010].

3. World Health Organization. International Agency for Research on Cancer. IARC monographs on the evalua- tion of carcinogenic risks to humans. Tobacco Smoke and Involuntary Smoking. Volume 83. 2004. Disponible en: http://monographs.iarc.fr/ENG/Monographs/vol83/ index.php [Consultado el 5 de Enero del 2010].

4. California Environmental Protection Agency. Air Resources Board. Proposed identification of environmental tobacco. Disponible en: http://www.arb.ca.gov/regact/ ets2006/ets2006.htm [Consultado el 2 de marzo de 2010].

5. Law M, Hackshaw A. Environmental tobacco smoke. $\mathrm{Br}$ Med Bull 1996; 52: 22-34.

6. López-Antunano F, Tovar-Guzmán V. Fumar o no fumar, en restoranes, hoteles y cantinas. Salud Publica Mex 2002; 44: S136-43.

7. Fichtenberg C, Glantz S. Effect of smoke-free workplaces on smoking behaviour: systematic review. BMJ 2002; 325(7357): 188.

8. Hammond S. Exposure of U.S. workers to environmental tobacco smoke. Environ Health Perspect 1999; 107: 329-40.

9. Wells A. Lung cancer from passive smoking at work. Am J Public Health 1998; 88: 1025-9.

10. Stayner L, Bena J, Sasco A, Smith R, Steenland K, Kreuzer $\mathrm{M}$, et al. Lung cancer risk and workplace exposure to environmental tobacco smoke. Am J Public Health 2007; 97: 545-51.

11. He J, Vupputuri S, Allen K, Prerost M, Hughes J, Whelton P. Passive smoking and the risk of coronary heart disease--a meta-analysis of epidemiologic studies. N Engl J Med 1999; 340: 920-6.

12. Steenland K. Risk assessment for heart disease and workplace ETS exposure among nonsmokers. Environ Health Perspect 1999; 107: 859-63.

13. Jaakkola M, Piipari R, Jaakkola N, Jaakkola J. Environmental tobacco smoke and adult-onset asthma: a population-based incident case-control study. Am J Public Health 2003; 93: 2055-60.

14. Mcghee S, Hedley A, Ho L. Passive smoking and its impact on employers and employees in Hong Kong. Occup Environ Med 2002; 59: 842-6.

15. Mcghee S. Passive smoking at work: the short-term cost. J Epidemiol Community Health 2000; 54: 673-6.

16. Ley $\mathrm{N}^{\circ} 20.105$, que modifica la Ley $\mathrm{N}^{\circ} 19.419$ en materias relativas a la publicidad y el consumo del tabaco (Publicada en el Diario Oficial de 16 de mayo de 2006). Gobierno de Chile.

17. Decreto No 594. Reglamento sobre condiciones sanitarias y ambientales básicas en los lugares de trabajo (Publicado en el Diario Oficial de 29 de abril de 2000). Gobierno de Chile.

18. Erazo M, Iglesias V, Droppelmann A, Acuña M, Peru- 
Ambiente laboral y exposición a humo de tabaco - P. Aceituno et al

ga A, Breysse P, Navas-Acien A. Secondhand Tobacco Smoke in Bars and Restaurants in Santiago, Chile: Evaluation of a Partial Smoking Ban Legislation in Public Places. Tobacco Control 2010. In press.

19. Johnsson T, Tuomi T, Hyvärinen M, Svinhufvud J, Rothberg M, Reijula K. Occupational exposure of nonsmoking restaurant personnel to environmental tobacco smoke in Finland. Am J Ind Med 2003; 43: 523-31.

20. Hammond S. A diffusion monitor to measure exposure to passive smoking. Environ Sci Technol 1987; 21: 494-7.

21. Nebot M, López M, Gorini G, Neuberger M, Axelsson S, Pilali M, et al. Environmental tobacco smoke exposure in public places of European cities. Tob Control 2005; 14: 60-3.

22. Navas-Acién A, Peruga A, Breysse P, Zavaleta A, BlancoMarquizo A, Pitarque R, et al. Secondhand tobacco smoke in public places in Latin America, 2002-2003. JAMA 2004; 291: 2741-5.

23. Bergman TA, Johnson DL, Boatright DT, Smallwood KG, Rando RJ. Occupational exposure of nonsmoking nightclub musicians to environmental tobacco smoke. Am Ind Hyg Assoc J 1996; 57: 746-52.

24. Jaakkola M, Jaakkola J. Assessment of exposure to environmental tobacco smoke. Eur Respir J 1997; 10: 238497.

25. Jenkins R, Counts R. Occupational exposure to environmental tobacco smoke: results of two personal exposure studies. Environ Health Perspect 1999; 107: 341-8.

26. U.S. Department of Health and Human Services. The
Health Consequences of Involuntary Exposure to Tobacco Smoke: A Report of the Surgeon General. U.S. Department of Health and Human Services, Centers for Disease Control and Prevention, Coordinating Center for Health Promotion, National Center for Chronic Disease Prevention and Health Promotion, Office on Smoking and Health 2006. Disponible en: http://www. cdc.gov/tobacco/data_statistics/sgr/2006/index.htm [Consultado el 13 de enero de 2010].

27. Barrientos-Gutiérrez T, Valdés-Salgado R, Reynales-Shigematsu L, Navas-Acién A, Lazcano-Ponce E. Exposición involuntaria al humo de tabaco en lugares públicos de la Ciudad de México. Salud Publica Mex 2007; 49: S205-12.

28. Lambert W, Samet J, Spengler J. Environmental tobacco smoke concentrations in no-smoking and smoking sections of restaurants. Am J Public Health 1993; 83: 1339-41.

29. Cains T, Cannata S, Poulos R, Ferson M, Stewart B. Designated "no smoking" areas provide from partial to no protection from environmental tobacco smoke. Tob Control 2004; 13: 17-22.

30. Ducatman A, Mclellan R. Epidemiologic basis for an occupational and environmental policy on environmental tobacco smoke. J Occup Environ Med 2000; 42: 1137-41.

31. World Health Organization. Protection from exposure to second-hand tobacco smoke. Policy recommendations; 2007. Disponible en: http://whqlibdoc.who.int/publications/2007/9789241563413_eng.pdf [Consultado el 10 de enero de 2010]. 